\title{
La profilaxis con antibióticos para colocación de DIU no esta indicada en mujeres con bajo riesgo de contraer enfermedades de transmisión sexual.
}

Randomised controlled trial of prophylactic antibiotics before insertion of intrauterine devices.

Terri Walsh, David Grimes, Ron Frezieres. Lancet 1998 Abril 4; 351:1005-1008.

\section{Objetivo}

Evaluar si la profilaxis antibiótica antes de la inserción del dispositivo intrauterino (DIU) reduce la necesidad de removerlo durante los 90 días posteriores.

\section{Diseño}

Ensayo clínico randomizado, controlado con placebo; con tres meses de seguimiento.

\section{Lugar}

Se realizó en 11 clínicas de Los Angeles, California; EE.UU.

\section{Pacientes}

Participaron del estudio 1985 mujeres que solicitaron colocación de DIU. Tenían una edad media de 30 años y bajo riesgo de contraer enfermedades de transmisión sexual (ETS), según un autocuestionario. Previa colocación de DIU, a todas las participantes se les realizó cultivo para Neisseria gonorreae e inmunofluorescencia o ELISA para Chlamydia trachomatis.

\section{Intervención}

Las pacientes fueron randomizadas para recibir $500 \mathrm{mg}$ de azitromicina o placebo una hora antes de la inserción del DIU. Las visitas médicas, luego de la inicial se realizaron a las 6 semanas y a los 3 meses. Se recogieron los datos de síntomas ginecológicos en todas las consultas.

\section{Medición de resultados principales}

El objetivo primario fue evaluar la necesidad de remover el DIU por cualquier causa diferente de expulsión parcial. Definieron salpingitis como dolor pelviano, abdominal o anexal más uno de los siguientes criterios: fiebre mayor a 38 grados, contenido purulento en la cavidad peritoneal, absceso pelviano, test positivo para Chlamydia o Gonoco$\mathrm{co}$, recuento de leucocitos mayor a $10.000 / \mathrm{mm}^{3}$. Secundariamente se evaluaron las consultas por síntomas ginecológicos.

\section{Resultados}

La tasa de remoción de DIU dentro de los 90 días de colocado fue similar en ambos grupos: 3,8\% (35/918) en el grupo tratado con antibiótico y $3,4 \%$ (31/915) en el grupo placebo. El RR fue de 1,1 (IC 95\% $0,7-1,8)$. Las consultas médicas posteriores no difirieron en ambos grupos (media: 38 consultas/100 mujeres). Sólo una mujer de cada grupo tuvo salpingitis definida.

\begin{tabular}{lcc}
\hline Causa de Remoción del DIU & Placebo & Antibiótico \\
\hline Sangrado abundante & 10 & 11 \\
\hline Dolor abdominal & 6 & 8 \\
\hline Dolor uterino o anexal & 5 & 1 \\
\hline Razones personales & 1 & 3 \\
\hline Infección genital baja & 1 & 2 \\
\hline Perforación & 1 & 1 \\
\hline Salpingitis & 0 & 1 \\
\hline Embarazo & 0 & 1 \\
\hline Otras & 7 & 7 \\
\hline Total de remociones & 31 & 35 \\
\hline Total de expulsiones & 31 & 32 \\
\hline Permanecieron insertos & $853(93,2 \%)$ & $851(92,7 \%)$ \\
\hline
\end{tabular}

Conclusiones

La profilaxis con antibióticos previa colocación de DIU, no cambia el riesgo de infección genital en mujeres de bajo riesgo para ETS.

\section{COMENTARIO}

La enfermedad inflamatoria pelviana (EPI), en el 90-95\% de los casos, es causada por los mismos agentes de las ETS: Gonococo, Chlamydia y Micoplasma. El mecanismo de infección es a través de transmisión sexual o por procedimientos ginecológicos (biopsia endometrial, inserción de DIU). Los factores de riesgo para la EPI son: múltiples parejas sexuales, antecedentes de haber presentado EPI o una ETS, no usar anticonceptivos de barrera y ser adolescente. Estos factores de riesgo son, entre otros, contraindicaciones de la colocación del dispositivo intrauterino. Aún es motivo de controversia la necesidad de tratamiento previo con antibióticos para reducir el riesgo de EPI en las pacientes que escogen este método anticonceptivo. Este estudio se realizó con un diseño adecuado para contestar esta pregunta, y con un correcto cálculo de poder estadístico*; a pesar de que la frecuencia de eventos fue ligeramente inferior a la que los auto- res habían previsto. Incluyó pacientes de bajo riesgo para uso de DIU, muestra* representativa de la población* de mujeres que habitualmente usan este método anticonceptivo o no tienen contraindicaciones para su colocación. No se halló diferencias en el riesgo de salpingitis dentro de los 90 días de la inserción entre las pacientes que habían y las que no habían recibido antibióticos. Por otro lado hubo diferencia no significativa en cuanto a las mujeres que presentaron dolor pelviano: en el grupo antibiótico 0,7\% vs. $1,3 \%$ en el grupo placebo. Por el momento parece razonable no administrar profilaxis antibiótica antes de la colocación de un DIU en mujeres de bajo riesgo que tengan un examen ginecológico normal. Es de importancia recalcar que las mujeres multíparas con una sola pareja sexual no tienen riesgo aumentado de EPI, por la colocación de DIU.

*Ver Glosario

\section{Dra. Vilda Discasciatti}

Unidad de Medicina Familiar y Preventiva. Hospital Italiano de Buenos Aires. 